\title{
Point-of-Use Water Filtration for Arsenic: A Sustainable and Simple Solution in Resource-poor Settings
}

\author{
Stephen D. Passman \\ Graduate Research Assistant \\ Dept. of Environmental \& Occupational Health \\ Saint Louis University \\ College for Public Health \& Social Justice \\ spassman@slu.edu
}

\author{
Tyler J. White, MPH \\ Graduate Research Assistant \\ Dept. of Environmental \& Occupational Health \\ Saint Louis University \\ College for Public Health \& Social Justice \\ Twhite26@slu.edu
}

Roger D. Lewis, PHD, CIH

Professor and Chair, Department of Environmental \& Occupational Health;

Director, Environmental Health Research Laboratory (EHRL)

Saint Louis University

College for Public Health \& Social Justice

Saint Louis, MO 63104

Lewisrd@slu.edu

\begin{abstract}
Lack of access to potable drinking water due to contamination from harmful metals is one of the most pressing public health issues globally. In areas close to mining and smelting operations, significant contamination from arsenic and other harmful metals can compound contamination from harmful microorganisms in drinking water. In response to this dual hazard, this study tests a prototype design of an improved point-of-use water filter for resource-poor areas and exposed populations. This project builds upon previous research that has demonstrated the effectiveness of clay pot filters impregnated with silver in reducing coliform bacteria. A compatible attachment comprised of activated charcoal in the form of bone char was added to the clay pot filter with the purpose of enabling the combined filter system to remove arsenic. The experiment tested the designed attachment with source water having an arsenic concentration of 500 parts per billion (ppb) to investigate the combined filter's ability to reduce the arsenic to acceptable World Health Organization drinking water standards of 10 parts per billion or below. The results demonstrate a significant decrease in the arsenic concentration to below the $10 \mathrm{ppb}$ standard with the bone char attachment, as well as a decrease in the arsenic concentration with the clay pot filter alone. These results warrant further investigation into the potential for clay pot filters with a charcoal layer to reduce arsenic concentrations in drinking water and demonstrate a potential additional benefit in arsenic removal by utilizing inexpensive bone char material in conjunction with the clay pot filter.
\end{abstract}

Index Terms: Clay Pot Filters, Arsenic, Point-of-use (POU) Water Filtration, Bone Char 


\section{INTRODUCTION}

Globally, approximately 780 million people lack access to a consistent source of clean drinking water ${ }^{1}$. Much of this arises from insufficient or ineffective treatment methods to remove harmful microorganisms in the water. In addition, contamination of water from harmful metals, such as arsenic, presents a significant obstacle to the provision of safe drinking water ${ }^{2}$.

In areas such as Bangladesh, India, and several countries in Central and South America, arsenic is present naturally in soils, often resulting in dangerous levels in many groundwater sources and creating a major public health concern for exposed populations ${ }^{3}$. Furthermore, arsenic can be a concern as a by-product of industrial mining and smelting operations, potentially contaminating nearby surface waters ${ }^{4}$. Consumption of arsenic-contaminated drinking water can lead to various health concerns, including skin diseases, and an increased risk for negative birth outcomes for pregnant women, among others ${ }^{3,5}$. In addition, arsenic is labeled as a group 1 carcinogen by The International Agency for Research on Cancer (IARC) ${ }^{6}$. This highlights the urgency of reducing arsenic levels in drinking water, especially drinking water in certain resourcepoor areas that may also contain harmful microorganisms.

\section{BACKGROUND}

Previous research in the department has shown significant arsenic contamination of surface waters in the proximity of a lead smelting plant in Peru ${ }^{4}$. Arsenic in drinking water around La Oroya, Peru, where much of the work from our Department has been, contains As III ${ }^{7}$. In this and other cases of industrial operations near surface water used for drinking, the water may contain both arsenic and bacterial contaminants, presenting two hazards to the health of the communities, which often depend on a sole and limited water source ${ }^{8}$. Therefore, there is a need to improve treatment methods to provide affordable and sustainable filtration of both harmful microorganisms and harmful metals such as arsenic.

In resource-poor and rural areas where systemic treatment of drinking water is not feasible, point-of-use water filtration is often used to address the concern of bacterial contamination. Several different types of point-of-use water filters have been developed, using materials such as sand, coagulants, chlorine disinfectants, silver and charcoal to physically or chemically reduce the concentration of microbes from the water ${ }^{9}$. One common design is a clay pot filter impregnated with silver, for which studies have demonstrated a nearly $100 \%$ effectiveness rate for removal harmful bacteria from drinking water ${ }^{9,10}$. These clay pot filters are distributed by many non-governmental organizations and manufacturers around the world, which increase their appeal given that many people already have the filter ${ }^{11}$.

A limitation of these clay pot filters is that they do not effectively reduce the concentrations of harmful metals, such as arsenic, present in the water ${ }^{9}$. Therefore, alternative point-of-use filtration mechanisms have been developed solely for the treatment of metals, given that concentrations of harmful bacteria are often insignificant in deep groundwater sources ${ }^{12}$. One such treatment involves the use of activated charcoal, or charcoal treated with oxygen to increase the number of small pores and 
consequently the surface area ${ }^{13}$. An example of activated charcoal is bone char, which can be produced by heating the dense carbon material found in animal bones to high temperatures to create a promising filtration material for adsorption of metals to the surface of the pores ${ }^{14}$. While the composition of bone char demonstrates its potential to reduce concentrations of harmful metals in water, studies have shown varying success in the use of bone char in point-of-use filters ${ }^{13,14}$.

To explore the issue of harmful metal contamination in drinking water, this study focuses on the example of arsenic. Having been linked to skin diseases, some cancers, negative birth outcomes, and other health problems, arsenic represents a public health hazard and is found naturally in the soil of some areas where groundwater is used for drinking water ${ }^{3,5,6}$. In response to these concerns, the US Environmental Protection Agency and the World Health Organization recommend a maximum arsenic concentration in water of 10 parts per billion (ppb) ${ }^{2}$. Point-of-use water filtration mechanisms, such as the use of iron oxide, activated aluminum or reverse osmosis, have been developed to significantly reduce the concentration of arsenic in groundwater used for drinking water ${ }^{12}$. However, the materials necessary for these processes are not often widely available in resource-poor areas and are not all capable of removing harmful bacteria in addition to arsenic ${ }^{14}$. Surface waters, which commonly contain significant concentrations of harmful microorganisms, can also be contaminated with arsenic, as evidenced by previous research within the Department of areas in which industrial lead smelting operations have taken place in Peru. Many surrounding surface water samples contained arsenic concentrations well above the $10 \mathrm{ppb}$ reference value, and a few over $1,000 \mathrm{ppb}(100 \text { times the limit set by the US EPA and the WHO })^{4}$. Given the significant concentrations of harmful bacteria that are often present in surface waters, there is a need to explore point-of-use filtration options that incorporate inexpensive materials and reduce concentrations of both harmful metals and bacteria.

Building upon research demonstrating the efficacy of ceramic pot filters impregnated with silver for removing significant bacteria levels from source water, this project investigates the potential for a new design that utilizes bone char to remove harmful metals, using arsenic as a test indicator ${ }^{10,14,15}$. This prototype design includes an attachment that is intended to reduce arsenic levels at the point-of-use in an affordable and sustainable way. The central part of the arsenic-filtration mechanism in the attachment is a simple layer of bone char. Brimac ${ }^{\circledR}$ NSF/ANSI 61 Certified Bone Char was used, which is a charcoal made from bovine bones ${ }^{16}$. Bone char is ideal because it allows for rural areas to recycle animal bones for use in the household clay pot water filter. Currently, there is no published research on the effectiveness of this method in combination with the clay pot filters, using a novel design that allows for slow-drip filtration.

Given the wide distribution of clay pot filters infused with silver around the world, this research project uses these filters to minimize the cost and burden for the communities who would benefit from this dual filtration ${ }^{11}$. By utilizing existing and inexpensive resources, this project seeks to expand access to potable drinking water as a means of improving public health in areas where arsenic contamination may compound the concerns of high microbial contamination in water. This research is part of a broad focus that the Department has on providing safe and affordable drinking water in resource-poor areas. 
Graduate and undergraduate students have participated in the Department's research on point of use drinking water and marketing point of use water filters. Selection of the Filter Pure ${ }^{\circledR}$ filter (ceramic water filters) came after close collaboration with a manufacturer and distributor in the Dominican Republic that had high quality control and a sustainable model for distribution in the Dominican Republic. Unpublished studies by four biomedical engineering students at Saint Louis University found that this water filter had sufficient coliform reduction and water flow to be adopted for the current study using an arsenic removal addition. The current study came about when two graduate students, on their own initiative, in September, 2012, sought to expand the work of previous students by exploring how arsenic reduction could be addressed through a combination of a ceramic water filter plus carbon-rich bone-char. The graduate students did the entire project as part of an independent, service learning experience that was supported by their School's graduate assistantship funding. The motivation for arsenic reduction came about when both students worked on projects to provide potable water in the Andes Mountains of Peru and Ecuador, where mining was affecting many communities with arsenic contamination.

There were over four faculty members that assisted the students from time to time, with study design, chemistry consultation, statistical analysis, and project management. Reports on this work will be directed to the manufacturer of the ceramic water filters who will utilize this information in service to Haitian and Dominican communities. In addition, the results will be shared with academic partners of Saint Louis University working in Peru and Ecuador to identify and mitigate environmental contaminants from industrial mining and smelting operations. Many rural communities living near extractive operations are dependent on surface water sources for drinking and sanitation, which potentially exposes them to water that is contaminated with both harmful bacteria and elevated concentrations of arsenic. Thus, an inexpensive point-of-use filtration mechanism that filters both arsenic and bacteria can be beneficial in these communities. Although the learning experience was primarily one based in a laboratory, the students both had significant field experiences in the Andes mountains regions to relate their work to the difficulties of adapting this or other point of use water filters in remote and under resourced regions.

\section{OBJECTIVES}

- Conduct a preliminary study to test a prototype bone char attachment to a clay pot filter, with an infused charcoal layer and compare it to the clay pot filter alone for assessing the efficacy of the newly designed system in reducing the concentration of arsenic in drinking water.

- Expand upon the potential for activated charcoal in the form of bone char to reduce the concentration of arsenic in drinking water using a slow drip mechanism.

- Contribute to the scientific literature on options for point-of-use filtration of arsenic in drinking water, with special consideration for populations in resource-poor areas which may experience exposure to arsenic in surface water that is also contaminated with harmful bacteria. 


\section{METHODS}

Two Filterpure ${ }^{\circledR}$ ceramic pot filters, manufactured in the Dominican Republic, were used in this experiment. The attachment was placed between one of the filters and its corresponding water receptacle to follow the model design (Figure 2). In the comparison of these two filters, 25 trials were performed via the methods outlined below. After the initial 25 trials, the attachment was moved ("switched") to the other filter for eight additional trials to account for variability in performance of the two ceramic pots. The procedures were as follows:

- Use inorganic arsenic from Fisher Scientific that was an unspecified combination of Arsenic III and V in nitric acid to prepare contaminated water.

- Pipet calculated amount of arsenic solution into 5 Liters of water to obtain a concentration of 500 parts per billion (ppb), confirmed each time by a Hach EZ arsenic test strip. The concentration of $500 \mathrm{ppb}$ was used to reflect a high level of arsenic exposure, 50 times reference limit set by the US EPA and the WHO. In the previous Department research in Peru, levels even above 1,000 ppb were found in some surface waters near smelting operations. Recognizing the lower frequency of these extreme values, $500 \mathrm{ppb}$ was chosen as a more realistic, while still significantly elevated, concentration of arsenic to which a person might be exposed in situations for which this filter is designed.

- Pour 5 Liter solution of contaminated water into both the control (clay filter) and the test filter (our lab model with bone char attachment) and allow water to filter for approximately 24 hours.

- Run 25 comparison trials using first setup in main experiment, then switch the clay filters for eight additional crossover trials to account for variability in clay pots

- Measure arsenic concentration before and after filtration using Hach EZ Arsenic Testing Kits and record date, time, and arsenic level in parts per billion.

- Verification of test strips (using EPA 200.8: ICP/MS for trace metals in water $)^{17}$ :

○ Take $100 \mathrm{~mL}$ aliquots for 9 control and 9 test filter final Arsenic concentrations that had been estimated with the Hach testing strips. Also take an aliquot with an initial, known concentration for a positive control.

- Send to Peoria Disposal Company (PDC) Laboratories, Inc. (3278 N. Hwy 67, Florissant, MO, 63033) as "blind" samples (i.e., the laboratory did not know which samples were control and which were test filters).

- Request mass spectrometry analysis for obtaining an accurate approximation of the arsenic concentrations. 


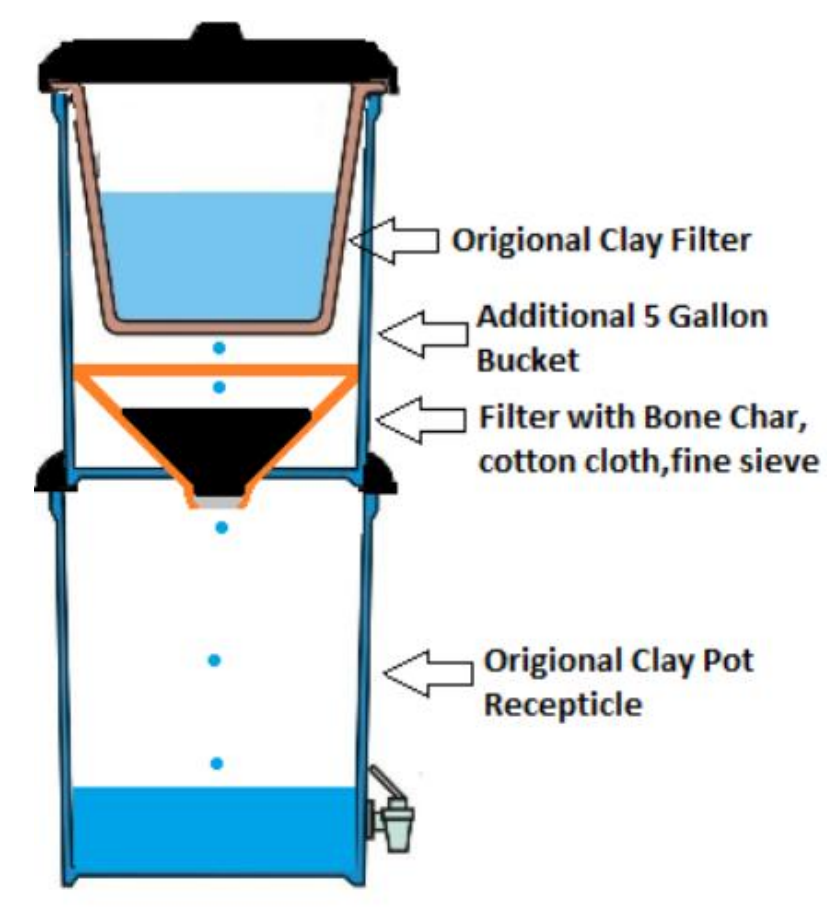

FIGURE 1

PROTOTYPE DESIGN

Figure 1 displays the initial attachment and original clay filter concept design. The attachment used minimal and easily-obtained resources and was simple to construct. This enables communities to make their own combination filters, without needing to import all new materials. This simple design has the potential to be replicated locally, promoting positive local economic impact in addition to better health outcomes through arsenic filtration. 

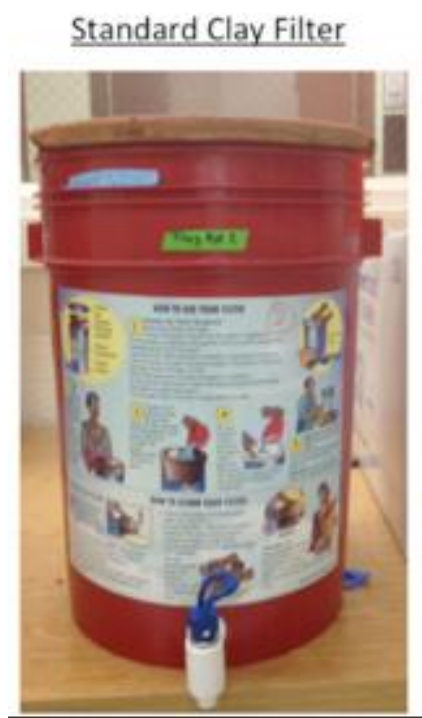

Designed Test Model (Bone Char Layer Plus Clay Filter)

Original

Clay Filter

EHRL Designed

Attachment

(Interior Bone

Char Layer)

Original Water

Receptacle

\section{FIGURE 2}

\section{STANDARD CLAY FILTER VS. PROTOTYPE (WITH FILTER ATTACHMENT)}

Figure 2 demonstrates the two main components that the research laboratory used for testing. The images are shown to juxtapose the original clay pot filter with the prototype model that includes the attachment:

\section{ReSUltS}

Preliminary results show a significant advantage of the test filter to reduce arsenic concentrations over the clay pot filter. Results from the main experiment, consisting of 25 trials, show that the test filter reduced arsenic concentrations to below the WHO and EPA drinking water standards. The crossover experiment, in which the filters were switched for quality control, consisted of only eight total trials due to limited resources and time constrictions but showed similar results. 


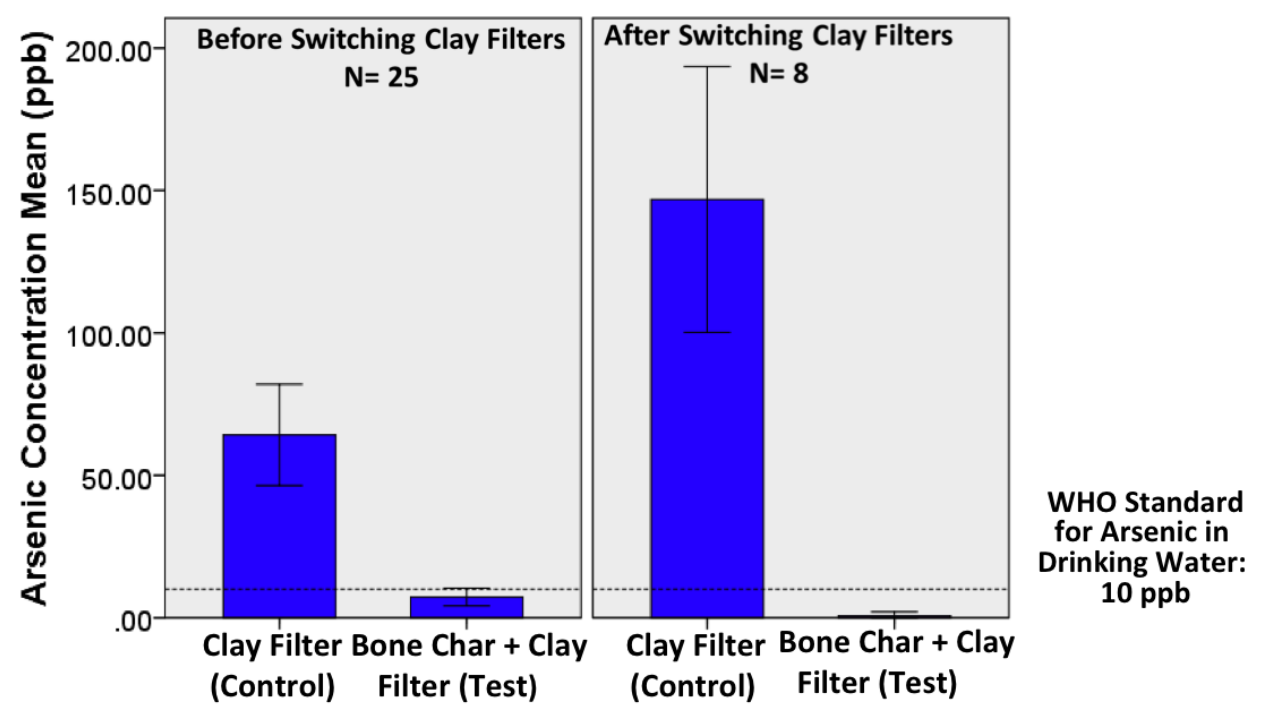

FIGURE 3

\section{POST-FILTRATION MEAN CONCENTRATIONS OF ARSENIC IN WATER}

Figure 3 summarizes the experimental results from the crossover study design by switching the bone char filter to the original control clay pot for increased quality assurance and quality control. The graph on the left depicts the arsenic concentrations that remained in post-filtration water, for test and control filters, in the original experiment with 25 trials. A ten-fold reduction in arsenic concentration was achieved in the control-ceramic pot filter with the combined filter and charcoal resulting in almost a 50 fold- reduction in arsenic concentration. The graph on the right depicts the postfiltration arsenic in water concentrations, for test and control filters, in the eight trials when the bone char attachment was moved from the test filter to the control. A three-fold reduction was achieved in the control ceramic pot filter with the combined filter and charcoal resulting in a near 100 -fold reduction of arsenic concentration, below the WHO standard for arsenic in drinking water. This "switch," making the control filter now the test filter and vice versa, was done to account for any variability that might exist in the composition of the clay filters themselves. As evident from the results, the switch, does account for some of the variability in the experiments. However, it is clear that while the ceramic pots represent significant reduction of arsenic concentration, ranging from a 3-10 fold reduction, the additional bone char contributes a 5-30 fold reduction in arsenic concentration. 


\section{Experiment Comparisons}

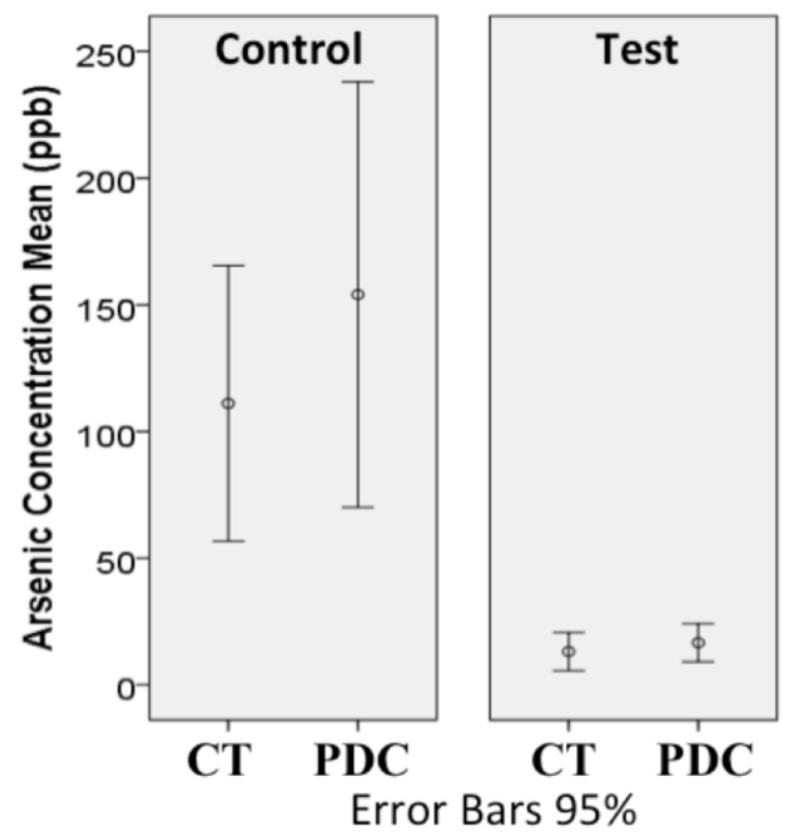

\section{CT $=$ Colorimetric Testing}

\section{PDC = PDC Laboratories verification, Method 200.8 Inductively Coupled Plasma Mass Spectrometry}

FIGURE 4

\section{EXPERIMENT CONCENTRATIONS COMPARING ICP- MS RESULTS AND HACH ARSENIC TESTING STRIPS (N = 9 FOR BOTH FILTERS)}

Figure 4 shows the results for the verification of accuracy of the testing strips utilized in the experimental methods, using inductively coupled plasma-mass spectrometry in a professional laboratory for comparison. As evidenced in these graphs, the results from testing strips were validated by laboratory analyses.

\section{DISCUSSION}

Both clay filters were used in the same experimental design to account for variability of filters and help quantify the influence of mineral composition of the clay pot filters. One of the major unexpected findings was that the clay pot filter actually filtered out a significant amount of arsenic, prompting further study into its composition and mechanism for filtration. Contrary to existing literature suggesting that clay pots typically do not filter harmful metals ${ }^{14}$, our results suggested that a ceramic pot filter can substantially reduce the amount of arsenic. After completion of the laboratory work for this study, a later report from students and professors, working in the Dominican 
Republic, reported that the ceramic water filters had a wood-based charcoal layer. This would help to explain the role of the ceramic pot in reducing arsenic concentrations in water. Results also suggest that the pots differ in their effectiveness, perhaps due to uniqueness and diversity in mineral content (especially charcoal composition) and variability in production.

Importantly, the results demonstrate that the clay filter with the bone char attachment showed the largest reduction in arsenic concentrations to below WHO and US Environmental Protection Agency (EPA) standards, when compared with the clay filter alone $^{2,6,12}$. This indicates that the added layer of activated carbon, in the form of bone char, has a beneficial effect on arsenic removal for point-of-use water filtration.

\section{Limitations \& STRENGTHS}

This study is a preliminary study with a limited number of replicates. As such, the project contains several limitations that are important to consider:

- Further testing is needed to ensure efficacy of arsenic removal over a longer period of time to estimate filter longevity. 25 trials at five gallons per trial were documented for results during the initial testing phase. Although the results showed consistent reduction in the concentration of Arsenic throughout this time period, further study is necessary to identify a realistic and practical time frame for the lifetime of the clay water filter and bone char attachment.

- This study used only two clay filters. It would be helpful to replicate the trials in additional filters to assess variation in the efficacy of reducing the concentration of arsenic in the water. Given the minor distinctions in the mineral content from one clay pot to another, additional replicates can help clarify the influence that filter composition has on overall efficacy of the structure.

- The arsenic solution is a combination of arsenic III and V, and thus our results do not differentiate between the two.

- Previous research has shown large variation in the flow rates of water through clay pot filters, which impacts the level of adsorption to the filter material ${ }^{10}$. This study did not consider flow rate as an independent variable. Flow rate was observed but no significant difference in the flow rate between the control and test filters (approximately $0.25 \mathrm{~L} / \mathrm{hr}$ ) was observed. However, further research should more thoroughly investigate the relationship of flow rate and arsenic removal in this system.

- This study did not examine Arsenic removal alongside removal of bacteria, given previous research showing the effectiveness of clay pot filters in removing coliform bacteria ${ }^{9}$. However, experimental testing of water contaminated with both Arsenic and coliform bacteria would help solidify the utility of the designed filter combination in areas where both contaminants are present.

- To assess the practicality of the filter, field research is needed that engages communities that could benefit from a simple POU filter that removes both harmful metals and harmful bacteria from drinking water. 
Despite these limitations, this study possesses certain strengths that are also important to highlight:

- Mass spectrometry verified the reliability of the Hach testing strips which were used to measure arsenic concentrations

- The design uses simple and widely available materials, which creates an opportunity to use local resources and expertise to maximize the sustainability of the filters.

\section{Conclusions}

The results suggest that the designed test filter has a significant potential for removing arsenic concentrations to below both WHO and EPA drinking water standards. In addition, the clay pot filter alone demonstrated substantial reduction in the concentration of arsenic. Further research must be done to investigate longevity and practicality of the test filter, and to explore the extent to which reduction in arsenic concentration is attributable to the additional bone char layer versus the clay pot filter.

Our research team aims to continue investigating the new design to maximize removal of arsenic, other harmful metals, and bacteria. Further development will also include finding the optimal pore size and surface area for maximum arsenic adsorption to the bone char layer and the clay pot itself. Given the variance in manufacture methods of different communities producing the clay pot filters, identifying physical characteristics that result in optimal arsenic adsorption can help promote best practices for improving the effectiveness of the filters. Finally, connecting with NGOs working in resource-poor communities is critical to improving POU clay pot water filters for enhanced filtration and better health outcomes.

This project is part of a broader effort by our researchers to protect the health of vulnerable populations using evidence-based practices to limit exposure to harmful contaminants in drinking water. The greater aim of this project is to connect this research to improve water quality and health outcomes, especially in rural and poor communities.

\section{ACKNOWLEDGMENT}

The faculty advisors on this project were Dr. Roger Lewis, Dr. Brett Emo, Dr. Christopher King, Dr. Kee-Hean Ong, Dr. Fernando Serrano, and Dr. Alan Zelicoff from the Department of Environmental and Occupational Health at the Saint Louis University College of Public Health and Social Justice. The authors appreciate the Saint Louis University Environmental Health Research Laboratory (EHRL) for the workspace and resources for this project. The authors also acknowledge the contributions of the previous EHRL research team of engineering students Muhammad Meigooni, John O’Donnel, and Stephanie Vernier. 


\section{REFERENCES}

1. Salaam-Blyther, T., Global access to clean drinking water and sanitation: US and international programs Congressional Research Service, Editor 2012.

2. Environmental Protection Agency, Basic informartion about the arsenic rule, 2012.

3. Yoshida, T., H. Yamauchi, and G. Fan Sun, Chronic health effects in people exposed to arsenic via the drinking water: dose-response relationships in review. Toxicology and Applied Pharmacology, 2004. 198(3): p. 243-252.

4. Serrano, F., Poison Harvest: Deadly U.S. Mine Pollution in Peru” - The Impact of Environmental Contamination on Public Health and Environmental Quality in La Oroya and the Mantaro Watershed, Hearing for Committee on Foreign Affairs of the US House of Representatives, Editor 2012.

5. Yang, C.-Y., et al., Arsenic in drinking water and adverse pregnancy outcome in an arseniasis-endemic area in northeastern Taiwan. Environmental Research, 2003. 91(1): p. 29-34.

6. World Health Organization, Exposure to Arsenic: A Major Public Health Concern, 2010.

7. Castro de Esparza, M.L. The presence of arsenic in drinking water in Latin America and its effect on public health. in International Congress: Natural Arsenic in the Groundwaters of Latin America. 2006. Mexico City: Pan American Center for Sanitary Engineering and Environmental Sciences.

8. Razo, I., et al., Arsenic and heavy metal pollution of soil, water and sediments in a semi-arid climate mining area in Mexico. Water, Air, and Soil Pollution, 2004. 152(1-4): p. 129-152.

9. Sobsey, M.D., et al., Point of use household drinking water filtration: A practical, effective solution for providing sustained access to safe drinking water in the developing world. Environmental Science \& Technology, 2008. 42(12): p. 42617.

10. Mwabi, J.K., B.B. Mamba, and M.N. Momba, Removal of Escherichia coli and faecal coliforms from surface water and groundwater by household water treatment devices/systems: A sustainable solution for improving water quality in rural communities of the Southern African development community region. International journal of environmental research and public health, 2012. 9(1): p. 139-170.

11. Potters for Peace. Ceramic Water Filter Project. 2013; Available from: http://pfp.he207.vps.webenabled.net/about-us/.

12. Kommineni, S., H. Durbin, and R. Narasimhan, Point-of-use, Point-of-entry Treatment for Arsenic Removal: Operational Issues and Costs2003: NSF.

13. Brunson, L.R. and D.A. Sabatini, An evaluation of fish bone char as an appropriate arsenic and fluoride removal technology for emerging regions. Environmental engineering science, 2009. 26(12): p. 1777-1784.

14. Mohan, D. and C.U. Pittman, Jr., Arsenic removal from water/wastewater using adsorbents--A critical review. J Hazard Mater, 2007. 142(1-2): p. 1-53. 
15. Mattelet, C., Household ceramic water filter evaluation using three simple lowcost methods: membrane filtration, $3 M$ Petrifilm and hydrogen sulfide bacteria in northern region, Ghana, 2006, Massachusetts Institute of Technology.

16. Services, B.C. Brimac Bone Charcoal. 2005; Available from:

http://www.sandia.gov/water/2005vendors/BrimacCarbonServices.pdf.

17. Environmental Protection Agency, Determination of Trace Elements In Water and Wastes By Inductively Coupled Plasma-Mass Spectrometry, in Revision 5.4, Methods for the Determination of Metals in Environmental Samples-Supplement I1994: EPA/600/R-94-111. 